\title{
Three-step priming in lexical decision
}

\author{
DOROTHEE J. CHWILLA and HERMAN H. J. KOLK \\ University of Nijmegen, Nijmegen, The Netherlands
}

\begin{abstract}
In two experiments, we investigated mediated two-step priming (e.g., from LION to STRIPES via TIGER) and three-step priming (e.g., from MANE to STRIPES via LION and TIGER). Experiment 1 showed robust twostep priming in the double lexical decision task. In Experiment 2, we tested for three-step priming and investigated the possibility that it is not association strength based on free association, but frequency of co-occurrence, that causes three-step priming. Co-occurrence has been proposed as a measure of familiarity and semantic relatedness. Significant three-step priming was obtained. Lexical co-occurrence could not account for the effect. However, a more global measure of semantic similarity that includes the similarity of the contexts in which concepts occur revealed that the three-step pairs were semantically related. If this global measure provides a proper estimate of the semantic relatedness of our items, then three-step priming is consistent not only with spreading activation models, but also with distributed memory models and the compound cue model.
\end{abstract}

Associative priming refers to the well-documented finding that a word (e.g., TIGER) is processed faster and/or more accurately when it is preceded by an associatively related word (e.g., LION) than when it is preceded by an unrelated word (e.g., RADIO). It is generally agreed that there are multiple causes or loci of associative priming (see Lucas, 2000, and Neely, 1991, for reviews). The focus in this article is on automatic priming. The traditional account of automatic priming is that it is caused by spreading activation (Anderson, 1983; Collins \& Loftus, 1975; Posner \& Snyder, 1975). Alternative accounts have evolved, however. The compound cue model of Ratcliff and McKoon (1988) and the distributed memory model of Masson (1991, 1995) have been presented as competitors of classic spreading activation models (for a discussion of spreading activation vs. compound cue models, see McKoon \& Ratcliff, 1992; McNamara, 1992a, 1992b, 1994; Ratcliff \& McKoon, 1988, 1994).

The goal of this article is to evaluate these models against each other by testing for mediated priming - that is, priming for target words that, according to free-association

Portions of this research were presented at the 38th Annual Meeting of the Psychonomic Society in Philadelphia, 1997. This research was supported by Grant 560-256-085 from the Netherlands Organization for Scientific Research (NWO). We are grateful to Timothy McNamara for his stimulating feedback on the very first plans to investigate mediated priming. We thank Michael Masson and Steve Joordens for valuable comments on a previous version of this paper. We are grateful to Rob Schreuder and Harald Baayen for giving us access to the Trouw corpus for computing the co-occurrence statistics. Finally, we thank Kathleen Jenks and Uli Chwilla for their assistance in analyzing the data. Correspondence concerning this article should be addressed to D. J. Chwilla, Nijmegen Institute for Cognition and Information, University of Nijmegen, P.O. Box 9104, 6500 HE Nijmegen, The Netherlands (e-mail: chwilla@nici.kun.nl). norms, are only indirectly related in semantic memory. In the case of one intervening concept, the facilitation is referred to as two-step priming (e.g., prime, LION; target, STRIPES; mediator [not presented], TIGER). In the case of two intervening concepts, the facilitation is referred to as three-step priming (e.g., prime, MANE; target, STRIPES; mediators [not presented], LION, TIGER).

Which of the three models can account for mediated priming and, if so, in what manner? Mediated priming is most straightforwardly explained by spreading activation models (Anderson, 1983; Collins \& Loftus, 1975). Since these models assume that activation spreads to all interconnected nodes, it should also spread from directly related concepts to associates of these concepts. In compound cue models, on the other hand (Ratcliff \& McKoon, 1988; for a similar proposal, see Dosher \& Rosedale, 1989), the target recognition is facilitated, not because it is preactivated, but because it represents, together with the prime item, a familiar combination (compound). These models can account for mediated priming only if they are combined with the appropriate kind of memory model. In combination with the search of associative memory model of Gillund and Shiffrin (1984), they predict an increase in familiarity for items related by one other item with high strength values to both the prime and the target. This implementation, thus, can account for two-step priming, but still not for three-step priming. Finally, distributed memory models (Masson, 1991, 1995; Sharkey \& Sharkey, 1992) explain all priming in terms of feature overlap/semantic similarity. In principle, therefore, distributed models can account only for one-step priming. If there is two-step or even three-step priming, this must mean that these pairs are somehow semantically related. If, indeed, mediated pairs were to turn out to be directly, although weakly, semantically related, then the compound cue model could account for these effects. 


\section{EVIDENCE FOR MULTISTEP PRIMING}

The first attempts to establish two-step priming in a standard lexical decision task were not successful (Balota \& Lorch, 1986; de Groot, 1983). McNamara and Altarriba (1988) argued that the absence of mediated priming might have been due to the presence of direct associates. These pairs could have invited participants to use relatednesschecking strategies, which might have masked mediated priming effects. Consistent with this idea, they demonstrated two-step priming when (1) a double lexical decision task was used with a list composed only of two-step pairs or (2) a single presentation lexical decision task was used that discouraged participants from using relatednesschecking strategies. Support for this hypothesis about the single-presentationlexical decision task comes from Shelton and Martin (1992), who observed two-step priming in this task, but not in a standard lexical decision task.

The results of Chwilla, Kolk, and Mulder (2000, Experiments 1 and 2) and de Groot (1983, Experiment 2), however, call into question the hypothesis that list composition plays a critical role in establishing two-step priming. In these three experiments using standard lexical decision, there was no indication for mediated priming, although the list included only two-step pairs. On the other hand, when Chwilla et al. (2000, Experiment 3) used the same materials in a double lexical decision task (which requires a lexical decision to both the primes and the targets), two-step priming occurred regardless of list composition-that is, not only in a pure list, but also in a mixed list. It is clear that the effects of list composition on mediated priming in lexical decision are not yet well understood. Although some results suggest that list composition is a decisive factor, other results suggest that omission of direct associates is not always effective in yielding two-step priming in standard lexical decision. Moreover, the observation of two-step priming in a mixed list in the double lexical decision task suggests that not list composition, but the requirement to judge the lexical status of both the prime and the target is crucial in bringing about mediated priming.

Chwilla and colleagues (2000) proposed that the mediated priming pattern in the double lexical decision task was most compatible with a spreading activation account. Yet one aspect of the data is not fully consistent with spreading activation models. Particularly, in the mixed list, the size of one-step and two-step priming was about the same (44 and $43 \mathrm{msec}$, respectively). Spreading activation models, however, predict a decay in priming with increasing distance in semantic memory. Therefore, one-step priming should have been larger than two-step priming. On the other hand, the results of McNamara and Altarriba (1988), in accordance with spreading activation models, indicate that in the single-presentation lexical decision task, onestep priming was larger than two-step priming (24 vs. $10 \mathrm{msec}$, respectively).

The goals of Experiment 1 were, first, to provide further evidence for the robustness of mediated priming in the double lexical decision task. If this task reliably evokes two- step priming, it may also be a sensitive tool to test for the theoretically more important three-step priming effects. A second goal was to investigate the size of one-step and two-step priming in the double lexical decision task. A decay in priming with associative distance would provide additional support for spreading activation models.

\section{EXPERIMENT 1}

\section{Method}

\section{Participants}

The participants were 30 undergraduates at the University of Nijmegen. They received course credit or were paid for their participation. All were native speakers of Dutch and had normal or correctedto-normal vision. Two participants had to be excluded from the analyses, one because of a history with reading problems and another because of an excessive number of errors $(>15 \%)$.

\section{Apparatus and Stimuli}

The participants were seated in a sound-attenuating chamber. A response device containing two push buttons was fixed on a small table in front of the participant. The stimuli consisted of 530 primetarget pairs that were presented in uppercase black letters on a white background at moderate contrast at the center of a PC monitor in a window of $8 \times 2 \mathrm{~cm}$. Three quarters of the primes and the targets were real Dutch words, and one quarter of the primes and the targets were nonwords. The nonwords were constructed in accordance with the phonotactic constraints of Dutch and were derived from real words by substituting 1 or 2 letters. Letter strings of 3-13 letters were presented as primes and targets.

The critical items were the same as those used by Chwilla et al. (2000). The free-association task was used to measure distance in semantic memory. The selection criterion for the two-step pairs was that the target did not occur as an associate of the prime in freeassociation norms (for details, see Chwilla et al., 2000). None of the items was repeated. The materials are available from the first author by e-mail.

\section{List Composition}

The pure list contained 100 two-step pairs (e.g., OORLOG-RUST, mediator VREDE; translation, WAR-QUIET, mediator PEACE) and 100 unrelated pairs (e.g., TOMAAT-RUST; translation, TOMATO-QUIET). Two versions were constructed in which the targets were counterbalanced across conditions and participants. Each version consisted of 50 two-step pairs and 50 unrelated pairs. Fifty unrelated filler pairs were added. This yielded a total of 150 word-prime-word-target pairs. Seventy-five pairs with a nonword prime and a word target and 75 pairs with a word prime and a nonword target were added to the list. The proportion of directly related word pairs, or relatedness proportion, was zero. The list was divided into two blocks of 150 pairs. Each block was preceded by 3 filler pairs.

The mixed list contained 49 one-step pairs (e.g., STORM-WIND; translation, STORM-WIND), 49 unrelated pairs (e.g., FAAM-WIND; translation, FAME-WIND), 39 two-step pairs (e.g., DAG-DONKER, mediator NACHT; translation, DAY-DARK, mediator NIGHT), and 39 unrelated pairs (e.g., DIJ-DONKER; translation, THIGH-DARK). The same target words were presented for the one-step pairs and their unrelated control pairs and for the two-step pairs and the unrelated pairs. The smaller number of two-step pairs than of one-step pairs was due to the fact that no more two-step pairs that fulfilled our selection criteria were available.

Two versions were constructed in which the targets were counterbalanced across conditions and participants. One version consisted of 25 one-step pairs, 24 unrelated pairs, 20 two-step pairs, and 19 unrelated pairs. The second version consisted of 24 one-step pairs, 25 unrelated pairs, 19 two-step pairs, and 20 unrelated pairs. Twenty-five 
unrelated filler pairs were added. This yielded a total of 113 word pairs. In the mixed list, the relatedness proportion was .22. Fifty-seven pairs with a nonword prime and a word target and 58 pairs with a word prime and a nonword target were added to the list.

\section{Procedure}

To avoid transfer of possible relatedness-checking strategies, the pure list always preceded the mixed list. Each trial began with the presentation of a fixation cross displayed at the center of the monitor. Five hundred milliseconds after the onset of the fixation cross, the prime and the target were presented simultaneously for $400 \mathrm{msec}$ (stimulus-onset asynchrony was $0 \mathrm{msec}$ ). The prime was presented left of the fixation cross, and the target was presented right of the fixation cross. The participants indicated by pressing a button with their dominant index finger that both letter strings were words or pressed a button with the other index finger to indicate that they were not. If they responded incorrectly, the Dutch word for error appeared on the screen for $2 \mathrm{sec}$. The percentage of correct yes and no decisions was $50 \%$. The participants were asked to respond quickly but, foremost, to respond accurately. A short practice list with 20 prime-target pairs preceded the experimental session.

\section{Results}

Because automatic priming models predict facilitation for related pairs, directional tests were carried out for a priori comparisons. Lexical decision times exceeding $2,300 \mathrm{msec}$ were removed from the analysis. All response times (RTs) more than 2.5 standard deviations above or below the mean for a participant or item within each condition were replaced by the mean of the means for that participant and item in that condition. Analyses were carried out on means computed for each participant or item and each condition. Mean RTs, standard deviations, and error rates for the two lists are presented in Table 1.

\section{Pure List}

Owing to a machine error, the last four trials of one version of the pure list were lost. These trials included two mediated items. The unrelated control pairs for these two items in the other version of the pure list were discarded from the analysis. The percentage of trials that fell outside the response boundaries, which was converted to the mean, was $0.58 \%(0.18 \%$ and $0.40 \%$ for the two-step and the control pairs, respectively). Mean response latencies were $41 \mathrm{msec}$ shorter for two-step pairs than for unrelated pairs. This difference was significant in the participant analysis $\left[t_{1}(27)=5.77, p<.001\right]$ and in the item analysis $\left[t_{2}(97)=\right.$ $3.30, p<.002]$.

The mean percentages of errors were $1.71 \%$ for the twostep pairs and $2.57 \%$ for the unrelated pairs. This difference in error rates was significant in the participant analysis $\left[t_{1}(27)=2.36, p<.02\right]$ and in the item analysis $\left[t_{2}(97)=\right.$ $1.73, p<.05]$. The error data showed the same pattern as that for the RT results, indicating that there was no speedaccuracy tradeoff.

\section{Mixed List}

The percentage of trials that fell outside the response boundaries, which was converted to the mean, was $2.40 \%$. For the one-step pairs, the percentage was $1.34 \%$ ( $0.65 \%$ for the related and $0.69 \%$ for the unrelated trials) and for the two-step pairs, $1.06 \%$ ( $0.53 \%$ for both the related and the unrelated pairs). Overall mean response latencies were shorter at distance one than at distance two $\left[F_{1}(1,27)=\right.$ $\left.19.08, p<.001 ; F_{2}(1,86)=5.88, p<.02\right]$ and for related than for unrelated items $\left[F_{1}(1,27)=24.74, p<.001\right.$; $\left.F_{2}(1,86)=9.73, p<.01\right]$. A priming effect was present for the one-step pairs $(82 \mathrm{msec})$, but not for the two-step pairs $(-5 \mathrm{msec})$. This interaction between distance and relatedness was significant in the participant and in the item analyses $\left[F_{1}(1,27)=55.00, p<.001 ; F_{2}(1,86)=12.43\right.$, $p<.001]$. Pairwise comparisons confirmed that a priming effect was present for the one-step pairs $\left[t_{1}(27)=\right.$ $\left.7.94, p<.001 ; t_{2}(48)=5.26, p<.001\right]$, but not for the two-step pairs (both $t \mathrm{~s}<1$ ).

The participant analysis of the error data did not yield effects of distance or relatedness (both $F_{1} \mathrm{~s}<2.5$ ) or an interaction between the two factors $\left(F_{1}<0.5\right)$. The item analysis showed a trend toward an effect of relatedness $\left[F_{2}(1,86)=3.02, p<.10\right]$. No distance $\times$ relatedness interaction was found $\left(F_{2}<0.05\right)$.

\section{Comparison of One-Step and Two-Step Priming}

Because two-step priming was obtained only in the pure list, we tested whether the one-step priming effect in the mixed list $(82 \mathrm{msec})$ differed from the two-step priming effect in the pure list $(41 \mathrm{msec})$. It was shown by $t$ tests on the difference between the one-step and the two-step priming effects that one-step priming in the mixed list was larger than two-step priming in the pure list $\left[t_{1}(27)=4.18\right.$, $\left.p<.001 ; t_{2}(145)=2.30, p<.02\right]$. A post hoc analysis revealed that the different sets of items were not matched in terms of word frequency. The mean word frequency for the one-step targets was significantly higher than that for the two-step targets. If the difference in the size of the priming effect was caused by word frequency, the largest priming effect should have been observed for the two-step items with a lower word frequency (e.g., Becker, 1979). Therefore, a frequency account of the difference in size in priming effects can be ruled out.

Table 1

Experiment 1: Mean Reaction Times (RTs, in Milliseconds) and Percentages of Errors (With Standard Deviations) for the Different Relatedness Conditions for the Pure List and the Two Levels of Distance for the Mixed List

\begin{tabular}{|c|c|c|c|c|c|}
\hline \multirow[b]{2}{*}{ Prime Type } & \multicolumn{2}{|c|}{ RT } & \multicolumn{2}{|c|}{ Errors } & \multirow[b]{2}{*}{ Priming Effect } \\
\hline & $M$ & $S D$ & $M$ & $S D$ & \\
\hline \multicolumn{6}{|c|}{ Pure List } \\
\hline Two-step & 861 & 125 & 1.71 & 1.40 & $41 *$ \\
\hline Unrelated & 902 & 124 & 2.57 & 2.18 & \\
\hline \multicolumn{6}{|c|}{ Mixed List } \\
\hline One-step & 768 & 102 & 2.43 & 5.15 & $82 *$ \\
\hline Unrelated & 850 & 103 & 4.43 & 5.37 & \\
\hline Two-step & 849 & 118 & 4.29 & 5.04 & -5 \\
\hline Unrelated & 844 & 120 & 5.18 & 5.69 & \\
\hline
\end{tabular}

$* p<.01$. 


\section{Comparison of Two-Step Priming in the Pure Versus Mixed List}

One might argue that the absence of two-step priming in the mixed list could be due to a lack of statistical power. We checked this possibility by conducting an analysis of variance (ANOVA) in which the two-step priming effects in the two lists were directly compared. This ANOVA revealed a list $\times$ relatedness interaction $\left[F_{1}(1,27)=22.92\right.$, $p<.001$ ], showing that the two-step priming effect in the pure list was significantly larger than that in the mixed list.

\section{Discussion}

The main results of Experiment 1 are as follows. First, clear two-step priming occurred in the double lexical decision task. This result bolsters the view that this task is a sensitive tool for investigating distance effects in semantic memory. Second, a list composition effect was found. Twostep priming occurred in a pure list, but not in a mixed list. The finding of a list composition effect in this task is consistent with the results of McNamara and Altarriba (1988) but is at odds with those of Chwilla et al. (2000) and de Groot (1983, Experiment 1). We take this effect to reflect that the participantsin the mixed list employed relatedness-checking strategies, which counteracted mediated priming. However, the discrepancies in results between studies indicate that the use of these strategies are not completely determined by list composition. Third, two-step priming was larger than one-step priming. This decay in priming with increasing associative distance is consistent with spreading activation models.

A more stringent test of spreading activation models against both alternative automatic priming models is the demonstration of three-step priming. Three-step priming is consistent with spreading activation models but cannot be explained by the various versions of the compound cue model. Distributed memory models also face serious difficulties in accommodating three-step priming, unless it can be shown that there exists some degree of semantic relatedness between the words. To date, there is only one report of three-step priming. McNamara (1992b) demonstrated three-step priming in the single-presentation lexical decision task. In light of the great theoretical implications of three-step priming for spreading activation models, it would be important to examine the reliability of this finding.

\section{EXPERIMENT 2}

The major goal in Experiment 2 was to test for three-step priming in the double lexical decision task in a list composed only of three-step pairs. The second aim in Experiment 2 was to investigate the possibility suggested both by McKoon and Ratcliff (1992) and by Masson (1991) that it is not association strength based on free-association norms but, rather, frequency of co-occurrence that determines (mediated) priming. Following McKoon and Ratcliff, we refer to frequency of co-occurrence as the frequency with which the prime and the target occur together in ordinary language, which can be estimated by the frequency with which the words co-occur in large corpora of text. Important in this context is that Masson (1995), arguing for a distributed model of priming, proposed co-occurrence frequency as a measure of semantic relatedness. The rationale underlying this proposal was that (1) the meaning of a concept is constructed from the context in which it occurs and that (2) concepts that frequently co-occur share many aspects of their contextually based meaning. This led Masson (1995) to predict that the frequency of co-occurrence in a language should be higher for semantically related words than for unrelated words. Therefore, if we match our material for frequency of co-occurrence and threestep priming is still observed, this will provide strong evidence for spreading activation models over alternative automatic priming models.

To test the reliability of the list composition effect, the pure list was followed by a mixed list including one-step and two-step pairs.

\section{Method}

\section{Participants}

The participants were 38 students who fulfilled the same criteria as those in Experiment 1. The data of 2 participants were rejected because of an excessive number of errors $(>15 \%)$.

\section{Apparatus and Stimuli}

The apparatus and presentation conditions were the same as those in Experiment 1. The stimuli consisted of 490 prime-target pairs. Three quarters of the primes and the targets were real Dutch words, and one quarter of the primes and the targets were nonwords. The nonwords were constructed in the same way as those in Experiment 1. Letter strings of 3-13 letters were presented as primes and targets. The materials are available from the first author by e-mail.

The three-step stimuli were based on the triplets of Experiment 1 (e.g., LION-[TIGER]-STRIPES). We generated words that were associatively related to the primes (e.g., MANE for LION). In this way we created 59 quadruplets. An example of a Dutch quadruplet is PEPER-[ZOUT]-[ZEE]-GOLVEN (translation, PEPPER-[SALT]-[SEA]WAVES). Free-association norms were collected from 74 undergraduates to verify that the presentation of the first word (e.g., PEPPER) produced the second word (e.g., SALT) but did not produce the third

Table 2

Experiment 2: Average Association Probabilities Between Words for the Three-Step Mediated Stimuli [e.g., Prime (MANe)-Mediator 1 (Lion)-Mediator 2 (TIGER)-Target (STRIPES)]

\begin{tabular}{lccc}
\hline & \multicolumn{3}{c}{ Association Probability } \\
\cline { 2 - 4 } Example Pair & Forward & Backward & Mean \\
\hline MANE-LION & .52 & .17 & .345 \\
MANE-TIGER & .003 & 0 & .0015 \\
MANE-STRIPES & 0 & 0 & 0 \\
LION-TIGER & .38 & .33 & .355 \\
LION-STRIPES & .0017 & .0022 & .00195 \\
TIGER-STRIPES & .40 & .48 & .44 \\
\hline
\end{tabular}

Note-The association probabilities are presented in both the forward and the backward direction (1) from the prime to Mediator 1, Mediator 2, and the target, respectively; (2) from Mediator 1 to Mediator 2 and the target, respectively; and (3) from Mediator 2 to the target. 
word (SEA) and/or the fourth word (WAVES) as an associate. In this and all the subsequent association tests, the participants were asked to produce five associates. Supplementary association norms from three groups of 45 undergraduates each were collected for the second word (first mediator), the third word (second mediator), and the fourth word (target). The data from these norms were combined with the free-association data collected for the primes. This yielded a total number of between 950 and 1,000 responses to the four words for each quadruplet. The average association probabilities between the words are presented in Table 2. A quadruplet was rejected if there was evidence for an associative shortcut - that is, if out of all responses to the four words, a mediator showed up as a direct associate more than two times. We considered these responses as too idiosyncratic to affect the results. On the basis of this criterion, 12 items had to be rejected. This yielded a total number of 47 three-step pairs.

\section{List Composition}

The pure list contained 47 three-step pairs (e.g., PEPER-GOLVEN; translation, PEPPER-WAVES; mediators, SALT and SEA) and 47 unrelated pairs (e.g., BLAFFEN-GOLVEN; translation, TO BARK-WAVES). Two versions were constructed in which the targets were counterbalanced across conditions and participants, so that no participant saw a word more than once. One version consisted of 24 three-step pairs and 23 unrelated pairs. The other version consisted of 23 threestep pairs and 24 unrelated pairs. In addition to the critical set of items, the list included 12 three-step pairs, which were excluded from the analysis on the basis of the supplementary association norms to the mediating words and the targets, and 70 unrelated filler pairs. This yielded a total of 129 word-prime-word-target pairs. One hundred twenty-nine pairs in which either a word target was preceded by a nonword prime (66 pairs) or a word prime was followed by a nonword target (63 pairs) were added to the list. The percentage of correct yes and no responses was $50 \%$.

The composition of the mixed list and the majority of the items was the same as that in Experiment 1. The changes were as follows. For the one-step pairs, 2 directly related pairs were replaced by other items. For the two-step pairs, 14 of the 39 two-step pairs were replaced by other pairs, because one of the items occurred as a prime or as a target of the three-step pairs. These two-step pairs were taken from the pure list of Experiment 1 .

\section{Procedure}

The procedure was identical to that in Experiment 1.

\section{Results}

The data trimming procedure was the same as that in Experiment 1. The results are presented in Table 3.

\section{Pure List}

Analyses based on free-association norms. The percentage of trials that fell outside the response boundaries and that was converted to the mean was $0.60 \%$ ( $0.30 \%$ for both the three-step and the control pairs). Mean response latencies were $935 \mathrm{msec}$ for the three-step pairs and $968 \mathrm{msec}$ for the unrelated pairs. This $33-\mathrm{msec}$ difference was significant in the participant analysis $\left[t_{1}(35)=3.58, p<.005\right]$ and in the item analysis $\left[t_{2}(1,46)=1.68, p=.05\right]$. These analyses indicate, therefore, that three-step priming was obtained.

The participants made fewer errors on three-step pairs $(2.78 \%)$ than on unrelated pairs $(3.55 \%)$. The difference in error rates was significant in the item analysis $\left[t_{2}(46)=\right.$ $1.83, p<.05]$, but not in the participant analysis $\left[t_{1}(35)=\right.$ $1.33, p<.10]$. The pattern of results resembled that of the RT data, showing that there was no speed-accuracy tradeoff.

Strict selection based on free association. One criticicism might be that we included items in the analyses for which a mediator showed up once or twice. The fact that the mediator does occur in free association might indicate that these words are directly related. The three-step priming effect might be caused by this subset of items. To check this possibility, we selected only those items in which all nonsuccessive associations were zero-that is, not a single participant listed the third word or the fourth word in response to the first (or vice versa) or the fourth word in response to the second (or vice versa) - and for which no mediating concepts could be found in the lists of associates collected for the primes and the targets (i.e., the first and the fourth words). These criteria were satisfied by 29 items. The analysis of this subset of items revealed that mean RT was shorter for the three-step items than for the control items $\left[t_{1}(35)=3.73, p<.005 ; t_{2}(28)=1.75, p<.05\right]$.

Analyses based on co-occurrence frequency. In the literature, the existence of true mediated priming has been questioned by Masson (1991) and by McKoon and Ratcliff (1992). Both suggested that mediated pairs may be directly but weakly related. Free association might not be sensitive enough to reveal these weak direct relationships. According to these researchers, the frequency with which

Table 3

Experiment 2: Mean Reaction Times (RTs, in Milliseconds) and Percentages of Errors (With Standard Deviations) for the Different Relatedness Conditions for the Pure List and the Two Levels of Distance for the Mixed List

\begin{tabular}{|c|c|c|c|c|c|}
\hline \multirow[b]{2}{*}{ Prime Type } & \multicolumn{2}{|c|}{ RT } & \multicolumn{2}{|c|}{ Errors } & \multirow[b]{2}{*}{ Priming Effect } \\
\hline & $M$ & $S D$ & $M$ & $S D$ & \\
\hline \multicolumn{6}{|c|}{ Pure List } \\
\hline Three-step & 935 & 119 & 2.78 & 2.73 & $33 *$ \\
\hline Unrelated & 968 & 125 & 3.55 & 3.26 & \\
\hline \multicolumn{6}{|c|}{ Mixed List } \\
\hline One-step & 798 & 135 & 2.22 & 3.37 & $73 *$ \\
\hline Unrelated & 871 & 132 & 5.22 & 4.46 & \\
\hline Two-step & 875 & 148 & 3.19 & 4.50 & $32 *$ \\
\hline Unrelated & 907 & 143 & 5.42 & 5.90 & \\
\hline
\end{tabular}

$* p<.01$. 
the primes and the targets co-occurred in the past, which can be estimated by the frequency of co-occurrence in large samples of written language, would be a better indicator of such weaker direct relationships.

To address this issue, we assessed the frequency of cooccurrence of the primes and the targets of the three-step pairs and the control pairs from a sample of about 5 million words taken from Trouw, a daily national Dutch newspaper. Our procedure was similar to that of McKoon and Ratcliff (1992). We computed the association ratio developed by Church and Hanks (1989), defined for two words $x$ and $y$ as the mutual information (unidirectional) between the two words- $\log 2[P(x, y) / P(x) P(y)]$. For a sample of language, this ratio compares the probability of observing the words $x$ and $y$ together (joint probability) with the probability of observing each of the words independently. If the two words are likely to co-occur in the sample, their joint probability will be larger than the product of their independent probabilities, and the value of the ratio will be larger than 1 . The probabilities were estimated from samples of Trouw. First, the independent probabilities for $x$ and $y$ were estimated by counting the number of times that the prime and the target occurred in the sample and normalizing by the number of words in the sample. Second, the joint probability of $x$ and $y$ was estimated by counting the number of times that the prime and the target were presented together within a window of 13 words, with the critical word in the 7 th position, with 6 words preceding and 6 words following the critical word, respectively.

First, to verify that the items were actually included in the corpus, we determined the frequency with which the primes and the targets of the critical pairs occurred independently. With the exception of two primes, all the words occurred in the corpus. The mean frequency of occurrence of the primes in this corpus was $126.85(S D=157.82)$, and the mean frequency of occurrence of the targets was $434.30(S D=986.76)$. Second, the co-occurrence analyses disclosed that 5 of the three-step pairs and 6 of the control pairs co-occurred in the Trouw corpus. The mean association ratios for these 5 three-step pairs and 6 unrelated items were 6.16 and 9.31 , respectively. We performed the most conservative analysis to test whether co-occurrence underlies the three-step priming effect-that is, we removed all items that co-occurred in the corpus and the corresponding three-step or control items. This led to the rejection of 10 items ( 1 item co-occurred in both the mediated and the unrelated version). The analyses on the remaining 37 items that did not co-occur in the corpus disclosed that mean RT was shorter for the three-step items than for the unrelated items $\left[t_{1}(35)=4.66, p<.005\right.$; $\left.t_{2}(36)=2.00, p<.03\right]$. Therefore, we reject the hypothesis that co-occurrence gave rise to the three-step priming effect in the present study.

Analyses based on a more global measure of semantic similarity. There are, however, reasons to assume that local co-occurrence is too limited as a measure of semantic similarity. For instance, synonyms, such as road and street, have very similar meanings but rarely occur in the environment of one another. In response to such shortcomings, global measures of co-occurrence have been developed. These global measures indicate how often two words co-occur with the same set of other words. To test whether a measure of semantic similarity that takes into account similarity of contexts could explain the priming patterns in Experiments 1 and 2, we submitted our stimuli to one of these global measures: the latent semantic analysis (LSA; Landauer \& Dumais, 1997; Landauer, Foltz, \& Laham, 1998; available on the Internet at http://lsa.colorado. edu/; see Burgess \& Lund, 2000, for a related approach). Specifically, we translated the one-step, two-step, three-step, and respective control pairs and carried out a "pairwise (term to term) comparison," using the tasaALL space, which corresponds to the 1st-year college level. For this space, the LSA matrix is based on the occurrence of 92,409 unique terms in 37,651 contexts selected from "texts, novels, newspaper articles, and other information.” The single value decomposition of this space yields vectors with a maximum of 419 dimensions. For ambiguous words, we computed the similarity values for both the dominant and the subdominant meanings. Because separate analyses including dominant

Table 4

Mean Semantic Similarity Values (SSV) for the Experimental and Control Items for the Different Levels of Distance for Experiments 1 and 2

\begin{tabular}{|c|c|c|c|c|c|c|c|}
\hline \multicolumn{4}{|c|}{ Experiment 1} & \multicolumn{4}{|c|}{ Experiment 2} \\
\hline Condition & LSA SSV & $d f$ & $t_{2}$ & Condition & LSA SSV & $d f$ & $t_{2}$ \\
\hline Pure list & & & & Pure list & & & \\
\hline Two-step & 0.186 & 97 & $6.73^{*}$ & Three-step & 0.115 & 46 & $3.00 *$ \\
\hline $\begin{array}{l}\text { Two-step control } \\
\text { Mixed list }\end{array}$ & 0.082 & & & $\begin{array}{l}\text { Three-step control } \\
\text { Mixed list }\end{array}$ & 0.072 & & \\
\hline One-step & 0.400 & 48 & $7.63^{*}$ & One-step & 0.399 & 48 & $7.36^{*}$ \\
\hline One-step control & 0.107 & & & One-step control & 0.113 & & \\
\hline Two-step & 0.202 & 38 & $3.30 *$ & Two-step & 0.204 & 38 & $4.44 *$ \\
\hline Two-step control & 0.115 & & & Two-step control & 0.088 & & \\
\hline
\end{tabular}

Note-The values are based on the latent semantic analysis (LSA) model, the "pairwise comparison" application (term to term) using the tasaALL space (which corresponds to the 1st-year college level). The significance of the difference in SSVs between the experimental and the control items was assessed by $t$ tests. $d f$, degrees of freedom. $* p<.01$, two-tailed. 
versus subdominant meanings yielded the same pattern of results, we only present the results of the analyses including dominant meanings.

The results of the LSAs are summarized in Table 4. The analyses for Experiments 1 and 2 showed (1) that the mean semantic similarity values for the two-step pairs were larger than those for unrelated items, both for the pure list and for the mixed list, and (2) that the mean semantic similarity value for the one-step pairs was larger than that for the unrelated pairs. The most important finding was that the LSA for the pure list revealed that the mean semantic similarity value for the three-step pairs was significantly larger than that for the control pairs. The theoretical importance of this result is that a more global measure of semantic relatedness that, in contrast to simple lexical cooccurrence, embraces the similarity of contexts in which words occur can account for three-step priming in Experiment 2. This outcome is the more remarkable if we consider that we translated our items from Dutch to American English.

\section{Mixed List}

The percentage of trials that fell outside the response boundaries, which was converted to the mean, was $2.53 \%$. For the one-step pairs, the percentage was $1.61 \%(0.92 \%$ for the related and $0.69 \%$ for the unrelated trials), and for the two-step pairs, $0.92 \%$ were converted to the mean ( $0.47 \%$ for the related and $0.45 \%$ for the unrelated pairs). Overall, mean response latencies were shorter at distance one than at distance two $\left[F_{1}(1,35)=60.57, p<.001\right.$; $\left.F_{2}(1,86)=15.65, p<.001\right]$ and for related than for unrelated items $\left[F_{1}(1,35)=55.47, p<.001 ; F_{2}(1,86)=\right.$ $19.86, p<.001]$. A priming effect was present for the onestep pairs (73 $\mathrm{msec})$ and for the two-step pairs $(32 \mathrm{msec})$. The interaction between distance and relatedness was significant in the participant analysis $\left[F_{1}(1,35)=16.62, p<\right.$ $.001]$, but not in the item analysis, in which distance was a between-items factor $\left(F_{2}<1\right)$. Planned comparisons revealed that the priming effect was significant for the onestep pairs $\left[t_{1}(35)=7.22, p<.001 ; t_{2}(48)=4.18, p<.001\right]$ and for the two-step pairs $\left[t_{1}(35)=4.54, p<.001 ; t_{2}(38)=\right.$ $2.27, p<.016]$.

The analyses of the error data revealed effects of relatedness $\left[F_{1}(1,35)=10.89, p<.003 ; F_{2}(1,86)=9.06, p<\right.$ .004]. Collapsed across distance, the participants were more accurate on the related pairs than on the unrelated pairs. No distance $\times$ relatedness interaction was found $\left(F_{1}\right.$ and $\left.F_{2}<1\right)$.

\section{Comparison of One-Step and Two-Step Priming}

Comparisons are reported only when the different sets were matched in terms of mean word frequency. It was shown by $t$ tests for the participant-based analyses on the difference between the one-step $(73 \mathrm{msec})$ and the twostep $(32 \mathrm{msec})$ priming effects that one-step priming was larger than two-step priming $\left[t_{1}(35)=4.08, p<.001\right]$. This difference did not reach significance in the item analysis.

\section{Discussion}

The main result of Experiment 2 was the demonstration of three-step priming in a pure list in the double lexical decision task. The lexical decision times to the three-step pairs in the participant- and item-based tests were significantly faster than the lexical decision times to unrelated pairs. Previously, McNamara (1992b) established threestep priming in a pure list in the single-presentation lexical decision task. The present data show that three-step priming also occurs in the double lexical decision task. The generalization of three-step priming to a new set of materials in Dutch provides a more solid basis for three-step priming in lexical decision.

The second major finding was that the three-step priming effect could not be explained in terms of lexical cooccurrence, which has been claimed to be a method of measuring distance in semantic memory superior to free association (Masson, 1991; McKoon \& Ratcliff, 1992). Three-step priming occurred despite the use of very strict criteria based on the free-association method or the lexical co-occurrence method. This result supports the view that the three-step priming effect was caused by spreading activation and appears to be inconsistent with the proposal that three-step priming might be accounted for by familiarity (McKoon \& Ratcliff, 1992) or semantic relatedness (Masson, 1991). A very surprising outcome, however, was that a more global measure of semantic relatedness that takes into account the similarity of the contexts in which words occur (Landauer \& Dumais, 1997; Landauer et al., 1998) demonstrates that the three-step pairs are semantically related, although weakly. This means that three-step priming is compatible also with alternative automatic priming models (Masson, 1995; McKoon \& Ratcliff, 1992). We will come back to this finding in the General Discussion section.

A third result was that in Experiment 2, in contrast to Experiment 1, no effect of list composition was obtained. That is, in the mixed list, priming occurred not only for the one-step pairs, but also for the two-step pairs. We closely examined the materials to check whether we could find differences between the sets of items that might explain the discrepancy in results. However, the majority of the twostep items were the same as those in Experiment 1, and there was also nothing peculiar about the substituted items that might explain the difference. Furthermore, the procedures employed in Experiments 1 and 2 were exactly the same. Third, there was some, but relatively small, size difference between the pure lists (306 pairs in Experiment 1 vs. 266 pairs in Experiment 2). The conflicting results on the effects of list composition unveil that we do not yet have experimental control over the relatedness-checking strategies. Although the chance that participants adopt this strategy is larger when direct associates are included (McNamara \& Altarriba, 1988), it is clear that the presence of these pairs does not necessarily bring them to use this strategy. Apparently, there is space for variation in individual preferences. One factor that might have affected the 
choice of strategy was the content of the pure list (two-step pairs in Experiment 1 vs. three-step pairs in Experiment 2) that always preceded the mixed list. As we will discuss below, one could argue that owing to the more remote relationship between the three-step pairs than between the two-step pairs, the pure list in Experiment 2 was less prone to the use of strategies.

The results on the decay in priming with associative distance are somewhat inconclusive. In agreement with Experiment 1 and the results of McNamara and Altarriba (1988), one-step priming was larger than two-step priming. This finding supports spreading activation models. In Experiment 2 , however, no difference in the magnitude of priming was found between two-step and three-step pairs (32 and $33 \mathrm{msec}$, respectively). This result is inconsistent with spreading activation models that predict that three-step priming should be smaller than two-step priming. One account could be that the two-step pairs elicit more relatedness-checking strategies. Although a spreading activation type of analysis seems to preclude this (only single-step related items would elicit such strategies), from the point of view of the other models, semantic similarity varies on a continuum, and relatedness-checking strategies become less likely, the smaller the similarity. This would mean that for the twostep pairs there could be some strategy use and some reduction of the priming effect, whereas for the three-step pairs there would be little strategy use and, consequently, little reduction in priming effect. In this way, the strategy factor could bring the two priming effects close together in size. It should be noted, however, that our frequency analyses indicate that a frequency-based account of the size difference cannot be ruled out. In fact, the mean word frequency for the primes was significantly lower for the three-step items than for the two-step items. Also, there is the very real possibility that we are witnessing the effects of variability in the size of a relatively small priming effect.

\section{GENERAL DISCUSSION}

The main finding of this article is the demonstration of three-step priming in the double lexical decision task. This result is predicted by spreading activation models (Anderson, 1983; Collins \& Loftus, 1975), but not by alternative automatic priming models, unless the three-step pairs are somehow semantically related. We conducted two types of co-occurrence analyses to test whether the threestep pairs were related. First, simple lexical co-occurrence analyses of the three-step and the control pairs in a large corpus of written language indicated that the three-step pairs were not semantically related. Second, LSAs were conducted to determine whether a more global measure of semantic relatedness that, in addition to lexical cooccurrence, takes into account the similarity of contexts in which words occur would confirm this outcome. To our surprise, these supplementary analyses showed that our threestep pairs were weakly related semantically. If LSA provides a proper estimate of the semantic relatedness of the three-step items, this implies that distributed memory models (Masson, 1991, 1995) and compound cue models
(McKoon \& Ratcliff, 1992; Ratcliff \& McKoon, 1988) can account for the three-step priming effect obtained in Experiment 2.

One could question the validity of the global measure on at least one ground. LSA is based on American English. Thus, to compute the semantic relatedness, we had to translate our items from Dutch to American English. The question is whether these translations adequately captured what might be subtle but possibly important differences in word characteristics or meaning between the three-step and the control pairs. This critique holds not only for the three-step items, but also for all other items. Inspection of Table 4, however, suggests that, on the whole, there is a good correspondence between the semantic similarity values, on the one hand, and the distance as derived from freeassociation norms, on the other hand. Specifically, the similarity values for all three sets of two-step items are very similar to each other, but smaller than the similarity values for the two sets of one-step pairs. Likewise, the similarity values for the two-step pairs were larger than those for the three-step pairs. Regarding the critical three-step pairs, it is important to note that the degree of semantic similarity as derived from LSA for the three-step related pairs is comparable with that of the unrelated control pairs of the one-step pairs and the unrelated control pairs of the two-step pairs in the mixed list of Experiment 1. In other words, the three-step items are as semantically unrelated as the control pairs of the one-step or two-step pairs. Nevertheless, they are still more semantically related than the control pairs of the three-step pairs. Hence, the obtained threestep priming effect can be explained in terms of a very small but reliable difference in the degree of semantic similarity between the three-step pairs and this specific set of control items. It seems reasonable to conclude thatas far as LSA provides a proper measure of semantic similarity - the three-step priming effect in this article can be explained not only by spreading activation models, but also by distributed memory models and the compound cue model.

In this context, it has to be pointed out that the fact that our three-step pairs were semantically related according to LSA does not necessarily imply that the three-step pairs used by McNamara (1992b) were semantically related, nor does it imply that semantically unrelated three-step pairs cannot be constructed. In fact, Livesay and Burgess (1998) showed, using HAL, that the two-step primes and targets developed by Balota and Lorch (1986) and subsequently used by McNamara and Altarriba (1988) and by Shelton and Martin (1992) were less semantically related than appropriate control pairs. The conclusion one reaches about the semantic relatedness of mediated items therefore may depend on which model of semantic relatedness one uses. The challenge for future investigations of mediated priming will be to try to develop mediated primes and targets that are not semantically related according to HAL, LSA, or some other, similar model.

In the above, we have noted some inconsistencies in the priming pattern over experiments. Are these alternative automatic models more effective in accounting for these 
inconsistencies than are spreading activation models? No, they are not. These models cannot account for the presence versus absence of a list composition effect in Experiments 1 versus 2 . Because the mean semantic similarity values of the two-step pairs between experiments are very similar (0.202 vs. 0.204$)$ and both sets of two-step pairs were significantly more semantically related than the control pairs, the different sets should have produced two-step priming. Neither can they account for equivalent priming effects for the two-step and the three-step pairs, because the semantic similarity value for the former is larger than that for the three-step pairs. Therefore, the different automatic priming models are equally successful in accounting for effects of mediated priming but fail to explain the discrepancies in priming pattern between our two experiments.

The evidence that distributed memory models and compound cue models can account for three-step priming is new and at odds with the traditional view that mediated priming —in particular, three-step priming-provides strong evidence for spreading activation models. The present article furthermore suggests that LSA is a very sensitive measure for assessing more subtle differences in semantic relatedness between sets of items. Since this information is not readily available from other, more timeconsuming methods, such as free association and lexical co-occurrence, this new approach to word meaning might be an important tool for future research on word recognition. It would be interesting to find a way to theoretically link this more global measure of co-occurrence to the build up of associative strength between related items, as described in spreading activation models.

\section{REFERENCES}

Anderson, J. R. (1983). The architecture of cognition. Cambridge, MA: Harvard University Press.

Balota, D. A., \& LoRch, R. (1986). Depth of automatic spreading activation: Mediated priming effects in pronunciation but not in lexical decision. Journal of Experimental Psychology: Learning, Memory, \& Cognition, 12, 336-345.

BECKER, C. A. (1979). Semantic context and word frequency effects in visual word recognition. Journal of Experimental Psychology: Human Perception \& Performance, 5, 252-259.

Burgess, C., \& Lund, K. (2000). The dynamics of meaning in memory. In E. Dietrich \& A. Markman (Eds.), Cognitive dynamics: Conceptual and representational change in humans and machines (pp. 117-156). Hillsdale, NJ: Erlbaum.

Church, K., \& Hanks, P. (1989). Word association norms, mutual information, and lexicography. In Proceedings of the 27th Annual Meeting of the Association for ComputationalLinguistics (pp. 76-83). Vancouver: Association for Computational Linguistics.

Chwilla, D. J., Kolk, H. H. J., \& Mulder, G. (2000). Mediated priming in the lexical decision task: Evidence from event-related potentials and reaction time. Journal of Memory \& Language, 42, 314-341.

Collins, A., \& Loftus, E. (1975). A spreading activation theory of semantic processing. Psychological Review, 82, 407-428.
DE Groot, A. M. B. (1983). The range of automatic spreading activation in word priming. Journal of Verbal Learning \& Verbal Behavior, 22, 417-436.

Dosher, B. A., \& Rosedale, G. (1989). Integrated retrieval cues as a mechanism for priming in retrieval from memory. Journal of Experimental Psychology: General, 118, 191-211.

Gillund, G., \& Shiffrin, R. M. (1984). A retrieval model for both recognition and recall. Psychological Review, 91, 1-67.

Landauer, T. K., \& Dumais, S. T. (1997). A solution to Plato's problem: The latent semantic analysis theory of acquisition, induction, and representation of knowledge. Psychological Review, 104, 211-240.

Landauer, T. K., Foltz, P. W., \& Laham, D. (1998). Introduction to latent semantic analysis. Discourse Processes, 25, 259-284.

LivesAY, K., \& BuRGESS, C. (1998). Mediated priming in high-dimensional semantic space: No effect of direct semantic relationships or cooccurrence. Brain \& Cognition, 37, 102-105.

LuCAS, M. (2000). Semantic priming without association: A meta-analytic review. Psychonomic Bulletin \& Review, 7, 618-630.

Masson, M. E. J. (1991). A distributed memory model of context effects in word identification. In D. Besner \& G. W. Humphreys (Eds.), Basic processes in reading: Visual word recognition (pp. 233-263). Hillsdale, NJ: Erlbaum.

Masson, M. E. J. (1995). A distributed memory model of semantic priming. Journal of Experimental Psychology: Learning, Memory, \& Cognition, 21, 3-23.

McKoon, G., \& RAtCLIFF, R. (1992). Spreading activation versus compound cue accounts of priming: Mediated priming revisited. Journal of Experimental Psychology: Learning, Memory, \& Cognition, 18, 1155-1172.

McNamara, T. P. (1992a). Priming and constraints it places on theories of memory and retrieval. Psychological Review, 99, 650-662.

McNamara, T. P. (1992b). Theories of priming: I. Associative distance and lag. Journal of Experimental Psychology: Learning, Memory, \& Cognition, 18, 1173-1190.

McNamara, T. P. (1994). Priming and theories of memory: A reply to Ratcliff and McKoon. Psychological Review, 101, 185-187.

McNamara, T. P., \& Altarriba, J. (1988). Depth of spreading activation revisited: Semantic mediated priming occurs in lexical decision. Journal of Memory \& Language, 27, 545-559.

NeELY, J. H. (1991). Semantic priming effects in visual word recognition: A selective review of current findings and theories. In D. Besner \& G. Humphreys (Eds.), Basic processes in reading: Visual word recognition (pp. 264-336). Hillsdale, NJ: Erlbaum.

Posner, M. I., \& SNyder, C. R. R. (1975). Attention and cognitive control. In R. L. Solso (Ed.), Information processing and cognition: The Loyola symposium (pp. 55-85). Hillsdale, NJ: Erlbaum.

RatclifF, R., \& McKoon, G. (1988). A retrieval theory of priming in memory. Psychological Review, 95, 385-408.

Ratcliff, R., \& McKoon, G. (1994). Retrieving information from memory: Spreading-activation theories versus compound-cue theories. Psychological Review, 95, 385-408.

Sharkey,A. J. C., \& Sharkey, N. E. (1992). Weak contextual constraints in text and word priming. Journal of Memory \& Language, 31, 543572.

Shelton, J. R., \& Martin, R. C. (1992). How semantic is automatic semantic priming? Journal of Experimental Psychology: Learning, Memory, \& Cognition, 18, 1191-1210.

(Manuscript received July 17, 2000; revision accepted for publication September 10, 2001.) 